\title{
Levistilide A reverses rat hepatic fibrosis by suppressing angiotensin II-induced hepatic stellate cells activation
}

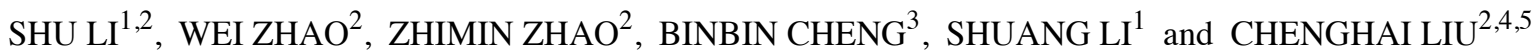

\begin{abstract}
${ }^{1}$ Department of Gastroenterology, Baoshan Branch, Shuguang Hospital Affiliated to Shanghai University of Traditional Chinese Medicine, Shanghai 201900; ${ }^{2}$ Institute of Liver Diseases, Shuguang Hospital Affiliated to Shanghai University of Traditional Chinese Medicine, Shanghai 201203; ${ }^{3}$ Department of Tradition Chinese Medicine, Changhai Hospital, Second Military Medical University, Shanghai 200433; ${ }^{4}$ Shanghai Key Laboratory of Traditional Chinese Clinical Medicine;

${ }^{5}$ Key Laboratory of Liver and Kidney Diseases, Ministry of Education, Shanghai 201203, P.R. China
\end{abstract}

Received December 21, 2018; Accepted March 26, 2020

DOI: $10.3892 / \mathrm{mmr} .2020 .11326$

\begin{abstract}
The renin angiotensin system (RAS) serves an important role in the development of hepatic fibrosis. Therefore, the present study investigated the effect of levistilide A (Lev A) on hepatic fibrosis via regulation of RAS. The effects of Lev A on the proliferation and activation of hepatic stellate cells (HSCs) were measured using a 5-ethynyl-2'-deoxyuridine assay, western blot analysis and immunofluorescence. The in vivo anti-hepatic fibrosis effect of Lev A was examined using a $\mathrm{CCL}_{4}$-induced rat fibrosis model. Lev A significantly prohibited angiotensin (Ang) II-induced proliferation of HSCs, and overexpression of smooth muscle $\alpha$-actin ( $\alpha$-SMA) and F-actin in HSCs. Lev A partly reversed Ang II-induced angiotensin type 1 receptor (AT1R) upregulation and ERK and c-Jun phosphorylation. In $\mathrm{CCL}_{4}$-induced hepatic fibrosis rats, $\mathrm{Lev} \mathrm{A}$ treatment significantly decreased the expression of collagen, $\alpha$-SMA and hydroxyproline in rat liver, and improved liver functions. Lev A treatment also significantly inhibited the $\mathrm{CCL}_{4}$-induced increase in plasma Ang II, and upregulation of AT1R and phosphorylated ERK in rat liver. In conclusion, Lev A is a potential agent for the treatment of hepatic fibrosis by suppressing Ang II/AT1R/ERK/c-Jun activation in HSCs.
\end{abstract}

Correspondence to: Professor Shuang Li, Department of Gastroenterology, Baoshan Branch, Shuguang Hospital Affiliated to Shanghai University of Traditional Chinese Medicine, 528 Zhangheng Road, Pudong New Area, Shanghai 201900, P.R. China

E-mail: lishuangbs@126.com

Professor Chenghai Liu, Institute of Liver Diseases, Shuguang Hospital Affiliated to Shanghai University of Traditional Chinese Medicine, 528 Zhangheng Road, Pudong New Area, Shanghai 201203, P.R. China

E-mail: chenghailiu@hotmail.com

Key words: angiotensin II, hepatic fibrosis, hepatic stellate cell, renin-angiotensin system, levistilide A

\section{Introduction}

Hepatic fibrosis is a risk factor in the progression of end-stage liver diseases, including cirrhosis and hepatocellular carcinoma. It is characterized by the progressive accumulation of extracellular matrix (ECM), such as smooth muscle $\alpha$-actin $(\alpha-S M A)$ and type I collagen (1). Hepatic stellate cells (HSCs) are a major source of ECM and serve important roles in hepatic fibrosis $(2,3)$.

Generally, the renin angiotensin system (RAS) is recognized as a manager of blood pressure and hydroelectrolyte balance. In recent years, accumulating evidence has demonstrated that RAS can also modulate the proliferation of HSCs, and the process of hepatic fibrosis via the profibrotic effector, angiotensin (Ang) II (4-6). The major biological actions of Ang II are mediated by the type 1 angiotensin receptor (AT1R); Ang II stimulates HSC migration, pro-collagen $\alpha 1$ expression, and the secretion of transforming growth factor (TGF) $\beta 1$ and inflammatory cytokines (7). In addition, blocking Ang II activity by lisinopril [an angiotensin-converting enzyme (ACE) inhibitor] or losartan (an AT1R antagonist) prevents the pro-fibrogenic effects of RAS $(8,9)$. In different models of hepatic fibrosis, including bile duct ligation, $\mathrm{CCl}_{4}$ treatment or continuous Ang II infusion, blocking the activity of Ang II by ACE inhibitor or AT1R antagonism also allivates hepatic fibrosis (10-12). Although angiotensinogen, which is a precursor for all angiotensin peptides, and its cleavage enzyme, renin, are not found to be elevated after liver injury, the levels of ACE and AT1R are markedly upregulated after liver injury, particularly in the fibrotic areas and activated HSCs $(13,14)$. These findings suggest that the RAS, especially Ang II, plays a vital role in liver fibrosis development, mainly through the activation of HSCs.

Traditional Chinese Medicine has been widely used in the treatment of a number of liver diseases. Angelica sinensis has been a commonly used medical herb in Traditional Chinese Medicine for thousands of years. It has been reported that Angelica sinensis can reverse high-fat diet-induced liver steatosis (15) and prevent $\mathrm{CCL}_{4}$-induced acute hepatic injury in rats (16). Levistilide A ( $\operatorname{Lev} A)$, the volatile oil extract of Angelica sinensis, inhibits HSC proliferation activated by 
platelet-derived growth factor BB by inducing cell cycle arrest and apoptosis (17). In preliminary experiments, Lev A also showed a significant effect on the proliferation and activation of HSCs induced by Ang II (data not shown). Therefore, the present study investigated the anti-hepatic fibrosis effect of Lev A on the the regulation of the RAS.

\section{Materials and methods}

HSC isolation and culture. A total of 10 normal male Sprague-Dawley rats (6-8 weeks old, $>400 \mathrm{~g}$ ) were obtained from The Shanghai Laboratory Animal Center, Chinese Academy of Sciences Shanghai, China. Rats were housed in a temperature controlled environment $\left(23 \pm 2^{\circ} \mathrm{C}\right)$, at a relative humidity of $60 \pm 10 \%$, with a 12-h light/dark cycle and free access to food and water. Primary HSCs were purified from Sprague-Dawley rats by perfusion with pronase and collagenase, followed by Nycodenz density-gradient centrifugation at $1,450 \mathrm{~g}$ for $22 \mathrm{~min}$ at $25^{\circ} \mathrm{C}$ (18). The isolated cells were cultured with Dulbecco's modified Eagle's medium (DMEM; Gibco; Thermo Fisher Scientific, Inc.) supplemented with $20 \%$ fetal bovine serum (FBS; Gibco; Thermo Fisher Scientific, Inc.). On the third day, the primary HSCs were used for subsequent experiments. The protocol was approved by the Committee on the Ethics of Animal Experiments of Shanghai University of Traditional Chinese Medicine, China. All animals received humane care during the study.

Cell proliferation analysis. HSCs were plated into 96-well plates and incubated at $37^{\circ} \mathrm{C}$ for $24 \mathrm{~h}$. Next, the cells were treated with $0.1 \mu \mathrm{M}$ Ang II (Sigma-Aldrich; Merck $\mathrm{KGaA})$ with or without Lev A (2.4, 6 and $15 \mu \mathrm{M}$; Shanghai Winherb Medical Technology Co., Ltd.,) or losartan (1 $\mu \mathrm{M}$; Sigma-Aldrich; Merck KGaA) for another $24 \mathrm{~h}$ at $37^{\circ} \mathrm{C}$. Cell proliferation was detected using the Cell-Light 5-ethynyl-2'-deoxyuridine (EdU) Cell Proliferation assay kit (cat. no. 10310; Guangzhou RiboBio Co., Ltd.) according to the manufacturer's protocols. The cell nuclei were stained with 4',6-diamidino-2-phenylindole (Sigma-Aldrich; Merck KGaA) at a concentration of $1 \mu \mathrm{g} / \mathrm{ml}$ for $10 \mathrm{~min}$ at room temperature. EdU-positive and EdU-negative cells were determined by fluorescent imaging. Images were taken using Cellomics ArrayScan VTI HCS Reader and analyzed using Cellomics Cell Health Profiling BioApplication software (version 3.1; Thermo Fisher Scientific, Inc.).

Immunofluorescence. HSCs were seeded into 96-well plates. After $24 \mathrm{~h}$, the cells were incubated with Ang II $(0.1 \mu \mathrm{M})$ with or without Lev A $(2.4,6$ and $15 \mu \mathrm{M})$ for another $24 \mathrm{~h}$ at $37^{\circ} \mathrm{C}$. The cells were then washed twice with cold phosphate-buffered saline (PBS) and fixed in 4\% paraformaldehyde for $10 \mathrm{~min}$ at $4^{\circ} \mathrm{C}$. F-actin and $\alpha$-SMA were determined by immunofluorescence staining as previously described (19). Images were captured using Cellomics ArrayScan VTI HCS Reader and analyzed using Cellomics Cell Health Profiling BioApplication software (Thermo Fisher Scientific, Inc.).

$\mathrm{CCL}_{4}$-induced hepatic fibrosis. A total of 24 male Wistar rats (6-8 weeks old; $240-260 \mathrm{~g}$ ) were obtained from the Shanghai Laboratory Animal Center, Chinese Academy of Sciences
Shanghai, China. The animals were housed with a $12-\mathrm{h}$ light/dark cycle under temperature control at $23 \pm 2^{\circ} \mathrm{C}$, with a relative humidity of $60 \pm 10 \%$ and free access to food and water. The rats were randomly assigned into 3 groups: Control group, model group and Lev A group. The animals in model and Lev A groups given intraperitoneal injections of $\mathrm{CCL}_{4}$ $(5 \mathrm{ml} / \mathrm{kg}$ ) for three consecutive days per week over a period of 4 weeks (20). From the third week of $\mathrm{CCL}_{4}$ injections, rats in the Lev A group were administered with Lev A at a dose of $3 \mathrm{mg} / \mathrm{kg} / \mathrm{day}$. Rats in the control and model groups were treated with an equal amount of vehicle. After 2 weeks of administration, rats were anaesthetized with $2 \%$ pentobarbital sodium and the blood samples were obtained from the inferior vena cava. A portion of each liver was fixed in $10 \%$ phosphate-buffered formalin for $24 \mathrm{~h}$ at room temperature for histological studies following paraffin embedding. The remainder was snap-frozen in liquid nitrogen and stored at $-80^{\circ} \mathrm{C}$ for western blot analysis. The protocol was approved by the Committee on the Ethics of Animal Experiments of Shanghai University of Traditional Chinese Medicine, China. All animals received humane care during the study.

Hematoxylin and eosin $(H \& E)$ and sirius red staining. The hematoxylin-eosin and sirius red staining were performed as previously described (21). Briefly, paraffin embedded-liver samples were cut into $4 \mu \mathrm{m}$-thick sections and then stained with $\mathrm{H} \& \mathrm{E}$ and sirius red. A random selection six microscopic fields were observed at a magnification of $\times 200$ with an inverted microscope (Mode: IX70, Olympus Corporation).

Serum biochemical measurements. Kits were used to measure levels of serum alanine aminotransferase (ALT; cat. no. C009-2-1), aspartate aminotransferase (AST cat. no. C010-2-1), albumin (Alb; cat. no. A028-2-1) and total bilirubin (TBil; cat. no. C019-1-1), according to the manufacturer's protocols (Nanjing Jiancheng Bioengineering Institute), as previously described (21).

Western blot analysis. Total protein was isolated from HSCs and liver tissue of rats using radioimmunoprecipitation assay buffer $(150 \mathrm{mM} \mathrm{NaCl}, 1 \%$ Nonidet P-40, $0.1 \%$ SDS, $50 \mathrm{mM}$ Tris- $\mathrm{HCl}$ pH 7.4, 1 mM EDTA, 1 mM PMSF, 1X Roche complete mini protease inhibitor cocktail, Roche PhosSTOP phosphatase inhibitor cocktail; Beyotime Institute of Biotechnology). The protein concentration was determined by the bicinchoninic acid method (Thermo Fisher Scientific, Inc.). Equal amounts of protein $(20 \mu \mathrm{g})$ were separated via SDS-PAGE on a $10 \%$ gel under denaturing and non-reducing conditions and then transferred to a nitrocellulose membrane. The membrane was blocked with odyssey blocking buffer (LI-COR Biosciences) at room temperature for $1 \mathrm{~h}$ and then incubated with primary antibodies against AT1R (1:200; cat. no. sc-1173; Santa Cruz Biotechnology, Inc.), phosphorylated (p)-ERK1/2 (1:200; cat. no. sc-7383; Santa CruzBiotechnology, Inc.), total ERK2 (1:200; cat. no. sc-1647; Santa Cruz Biotechnology, Inc.), GAPDH (1:5,000; cat. no. sc-166574; Santa Cruz Biotechnology, Inc.), $\alpha$-SMA (1:1,000; cat. no. ab5649; Abcam), c-Jun (1:1,000; cat. no. 9165; Cell Signaling Technology, Inc.) and p-c-Jun (1:1,000; cat. no. 9261; Cell Signaling Technology, Inc.) at $4^{\circ} \mathrm{C}$ overnight. Following washing in PBS with $0.1 \%$ Tween-20, 
A
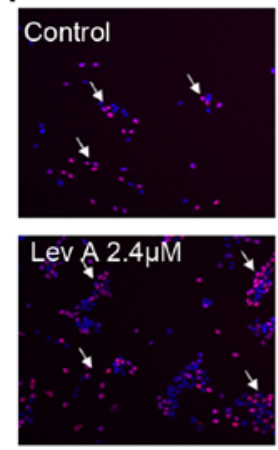
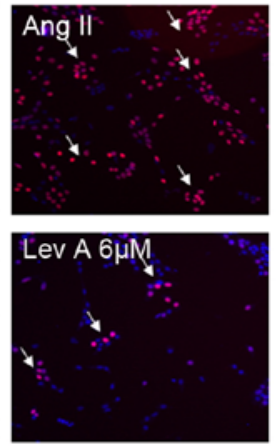

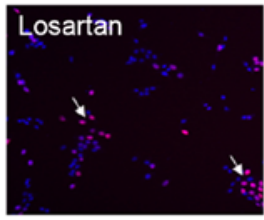

Lev A $15 \mu \mathrm{M}$

(1).
B

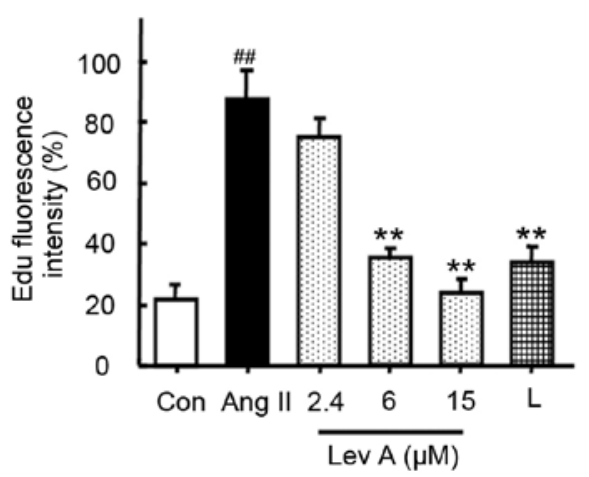

Figure 1. Lev A inhibits Ang II-induced HSCs proliferation. Following treatment with Ang II (0.1 $\mu \mathrm{M})$ and indicated concentrations of Lev A and losartan for $24 \mathrm{~h}$, cell proliferation was determined by the EdU method. (A) HSC proliferation was determined by EdU staining (magnification, x100; red, EdU; blue, DAPI). (B) Quantification of the fluorescence intensity. Each bar represents the means \pm standard deviation $(\mathrm{n}=6)$. ${ }^{\# \#} \mathrm{P}<0.01$ vs. control group; ${ }^{* *} \mathrm{P}<0.01$ vs. Ang II group. Lev A, levistilide A; Ang II, angiotensin II; HSCs, hepatic stellate cells; EdU, 5-ethynyl-2'-deoxyuridine; DAPI, 4',6-diamidino-2-phenylindole; L, losartan.

the blots were incubated for $1 \mathrm{~h}$ at room temperature with a IRdye ${ }^{\circledR} 680 \mathrm{CW}$ anti-rabbit (1:20,000; cat. no. 926-32223) or IRDye 800CW anti-mouse secondary antibody (1:20,000; cat. no. 926-32210), both purchased from LI-COR Biosciences. The signals were visualized and analyzed using Li-Cor Odyssey Imaging software (version 2.1; Li-Cor Biosciences).

Statistical analysis. Data are expressed as means \pm standard deviation, and were analyzed using one-way analysis of variance, followed by a post hoc least significant difference test. Statistical analysis was conducted using SPSS version 18.0 software (SPSS, Inc.). $\mathrm{P}<0.05$ was considered to indicate a statistically significant difference.

\section{Results}

Lev A inhibits HSC proliferation. After $24 \mathrm{~h}$ incubation, Ang II significantly increased the cell number of HSCs compared with the control, as determined by EdU assay $(\mathrm{P}<0.01$; Fig. $1 \mathrm{~A}$ and $\mathrm{B})$. Co-treatment with Lev A significantly decreased the number of HSCs proliferating at concentrations of 6 and $15 \mu \mathrm{M}(\mathrm{P}<0.01)$. However, $2.4 \mu \mathrm{M}$ of Lev A exhibited little effect on the proliferation Ang II-induced HSCs. The blocker of AT1R, losartan, also significantly suppressed the Ang II-induced proliferation of HSCs $(\mathrm{P}<0.01$; Fig. 1A and $\mathrm{B})$.

Lev A inhibits the activation of HSCs. To investigate the effect of Lev A on the activation of Ang II-induced HSCs, the expression of $\alpha$-SMA and F-actin was determined. Ang II treatment significantly enhanced the expression of $\alpha$-SMA $(\mathrm{P}<0.01)$, whereas co-treatment with losartan or Lev A significantly suppressed Ang II-induced $\alpha$-SMA upregulation $(\mathrm{P}<0.05$; Fig. 2A and B). Western blot analysis also demonstrated that Lev A inhibited Ang II-induced $\alpha$-SMA upregulation $(\mathrm{P}<0.01$; Fig. 2C and D). The present study also determined the expression of F-actin after Ang II treatment using immunofluorescence staining. Ang II induced a significant upregulation of F-actin ( $\mathrm{P}<0.01$; Fig. $2 \mathrm{E}$ and $\mathrm{F})$ and Lev A partly reversed the effect of Ang II on F-actin $(\mathrm{P}<0.05)$.
Lev A suppresses Ang II-induced ATIR upregulation and activation of ERK and c-Jun. Our previous study (21) revealed that Ang II is able to increase the level of its receptor AT1R in HSCs and so the effect of Lev A on AT1R expression was examined. Ang II treatment significantly upregulated the expression of AT1R ( $\mathrm{P}<0.01$; Fig. 3A and B). Losartan and Lev A treatment both repressed Ang II-induced AT1R upregulation $(\mathrm{P}<0.01)$. Activation of ERK and c-Jun is involved in Ang II-induced HSCs proliferation and activation $(12,21)$. Therefore, the effect of Lev A on the activation of ERK and c-Jun was also studied. Ang II treatment induced significant increases of $\mathrm{p}-\mathrm{ERK} 1 / 2$ and $\mathrm{p}$-c-Jun $(\mathrm{P}<0.01$; Fig. 3A, C and D). Pretreatment with Lev A significantly inhibited the phosphorylation of ERK1/2 and c-Jun induced by Ang II $(\mathrm{P}<0.05)$.

Lev A improves $C C L_{4}$-induced rat hepatic injury. To evaluate the in vivo effect of $\mathrm{Lev} \mathrm{A}$, a $\mathrm{CCL}_{4}$-induced rat hepatic fibrosis model was used. $\mathrm{CCL}_{4}$ significantly decreased the body weight, and increased the liver/body weight ratio $(\mathrm{P}<0.01$; Fig. 4A and B). Treatment with Lev A significantly improved the liver/body weight ratio $(\mathrm{P}<0.01)$ but it did not alter the body weight. In the model group, $\mathrm{CCL}_{4}$ treatment significantly decreased the albumin level and increased the ALT, AST, $\gamma-\mathrm{GT}$, IBIL and TBil levels $(\mathrm{P}<0.01$; Fig. 4C-H). Lev A treatment significantly improved the liver functions of experimental rats with hepatic fibrosis. $\mathrm{H} \& \mathrm{E}$ staining demonstrated that $\mathrm{CCL}_{4}$ induced evident centrilobular hepatic necrosis and infiltrating lymphocytes, and Lev A treatment partly prevented the histopathological changes (Fig. 4I).

Lev A ameliorates $\mathrm{CCL}_{4}$-induced liver fibrosis in rats. $\mathrm{CCL}_{4}$ treatment led to a notable deposition of collagen in rat livers as determined by sirius red staining (Fig. 5A). Treatment with Lev A for 2 weeks resulted in an alleviation of collagen deposition (Fig. 5A). As determined by western blotting, $\alpha$-SMA and collagen I expression was significantly increased in $\mathrm{CCL}_{4}$-treated rats and was suppressed by Lev A $(\mathrm{P}<0.01$; Fig. 5B-D). In $\mathrm{CCL}_{4}$-treated rats, the hydroxyproline (Hyp) content was also significantly upregulated compared with 

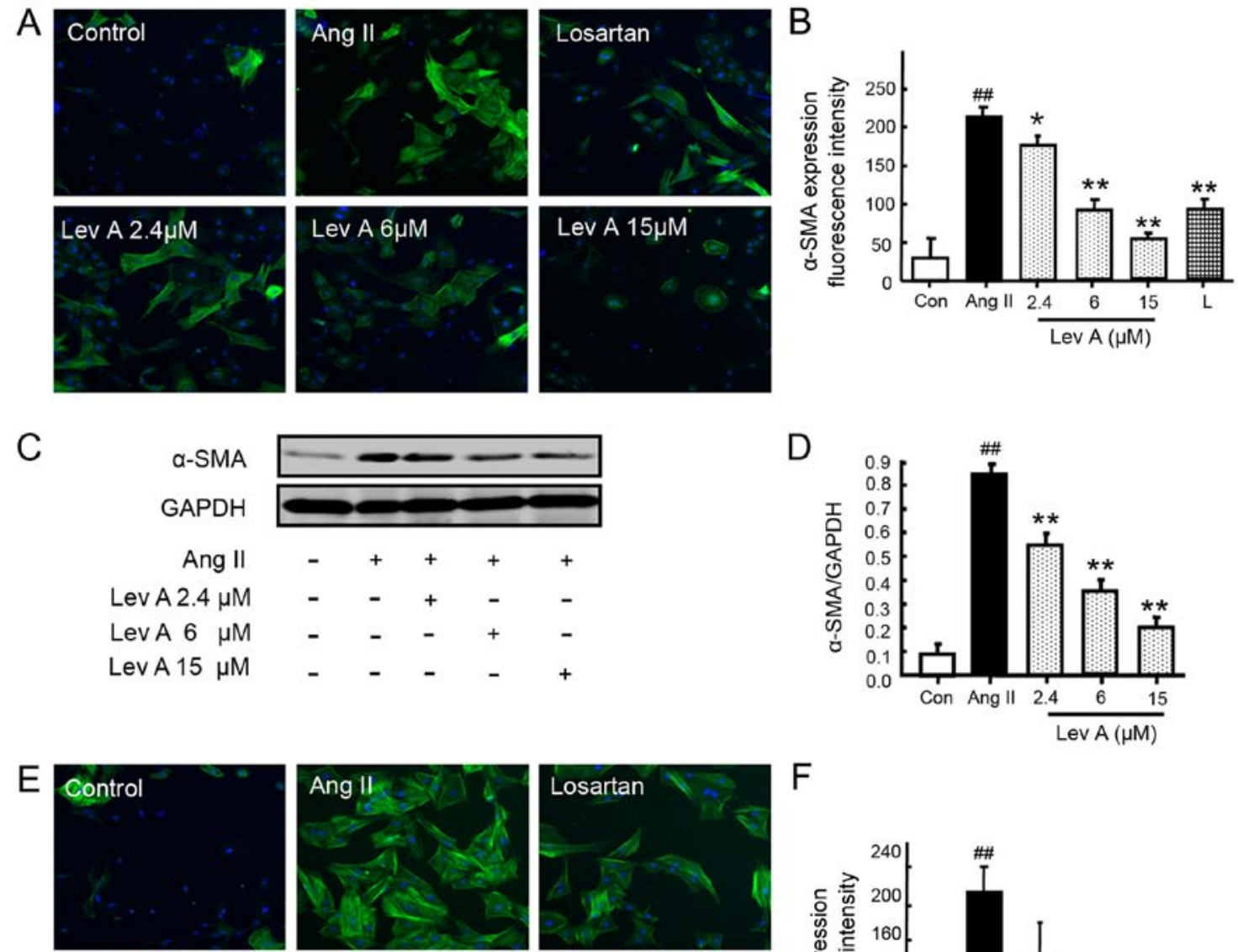

$\mathrm{D}$
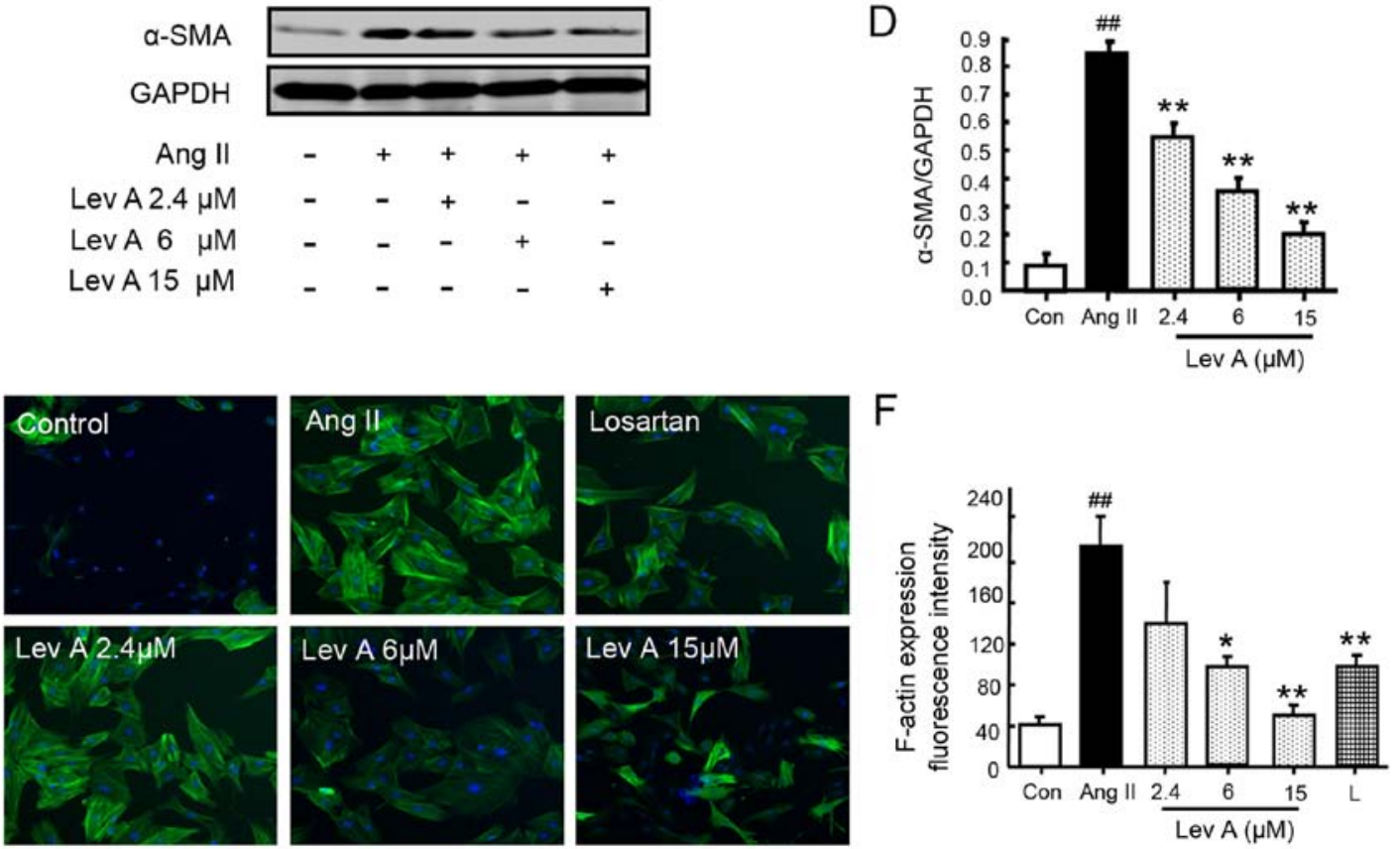

Figure 2. Lev A inhibits Ang II-induced HSC activation. (A) Immunofluorescence was used to determine the expression of $\alpha$-SMA. (B) Quantitative data of $\alpha$-SMA expression determined by immunofluorescence. (C) Westerbn blot analysis of the expression of $\alpha$-SMA and (D) the representative quantitative data. (E) The expression of F-actin was examined by immunofluorescence. (F) Quantitative data of F-actin expression. Each bar represents the means \pm standard deviation ( $\mathrm{n}=6) .{ }^{\# \#} \mathrm{P}<0.01$ vs. control group; ${ }^{*} \mathrm{P}<0.05,{ }^{* * *} \mathrm{P}<0.01$ vs. Ang II group. Lev A, levistilide A; Ang II, angiotensin II; HSCs, hepatic stellate cells; $\alpha$-SMA, smooth muscle $\alpha$-actin; L, losartan.

the control group $(\mathrm{P}<0.01$; Fig. 5E). Treatment with Lev A significantly decreased the Hyp content $(\mathrm{P}<0.01)$.

Lev A regulates the RAS signaling molecules in vivo. To further evaluate the role of Lev A on RAS in vivo, the molecules of ACE-Ang II-AT1R axis in rats were next determined. In the model group, the expression of AT1R was significantly upregulated compared with the control group ( $\mathrm{P}<0.01 ;$ Fig. $6 \mathrm{~A}$ and $\mathrm{B})$. p-ERK, the downstream molecule of Ang II-AT1R, was also significantly increased in the model group compared with the control $(\mathrm{P}<0.05$; Fig. 6A and $\mathrm{C})$. Lev A treatment significantly decreased the $\mathrm{CCL}_{4}$-induced increase of AT1R, Ang II and p-ERK $(\mathrm{P}<0.01)$. Compared with the control group, the plasma Ang II level was also significantly increased in the $\mathrm{CCL}_{4}$ group $(\mathrm{P}<0.01$; Fig. $6 \mathrm{D})$.

\section{Discussion}

The data from the present study demonstrated that Lev A was able to inhibit Ang II-induced proliferation and activation of HSCs. Lev A also suppressed Ang II-induced upregulation of AT1R and activation of ERK and c-Jun. In the $\mathrm{CCL}_{4}$ rat model, treatment with Lev A ameliorated liver fibrosis and improved liver function. In addition, Lev A also inhibited $\mathrm{CCL}_{4}$-induced increase of Ang II and AT1R and phosphorylation of ERK.

Ang II serves a vital role in the activation of HSCs and secretion of ECM (22). Therefore, numerous effective therapeutic approaches have focused on the RAS, particularly Ang II $(4,9)$. In accordance with previous studies $(6,23,24)$, Ang II stimulation promoted the proliferation of HSCs and upregulated the levels of $\alpha$-SMA and F-actin in HSCs. Lev A treatment inhibited Ang II-induced HSC proliferation and activation, indicating that Lev A may be an effective agent in the treatment of hepatic fibrosis. Subsequently, it was further investigated whether Lev A could also inhibit hepatic fibrosis in vivo. In the $\mathrm{CCL}_{4}$-induced rat hepatic fibrosis model, Lev A significantly inhibited the expression of $\alpha$-SMA and collagen, and improved the rat liver function, exhibiting a good therapeutic effect. These data suggested 

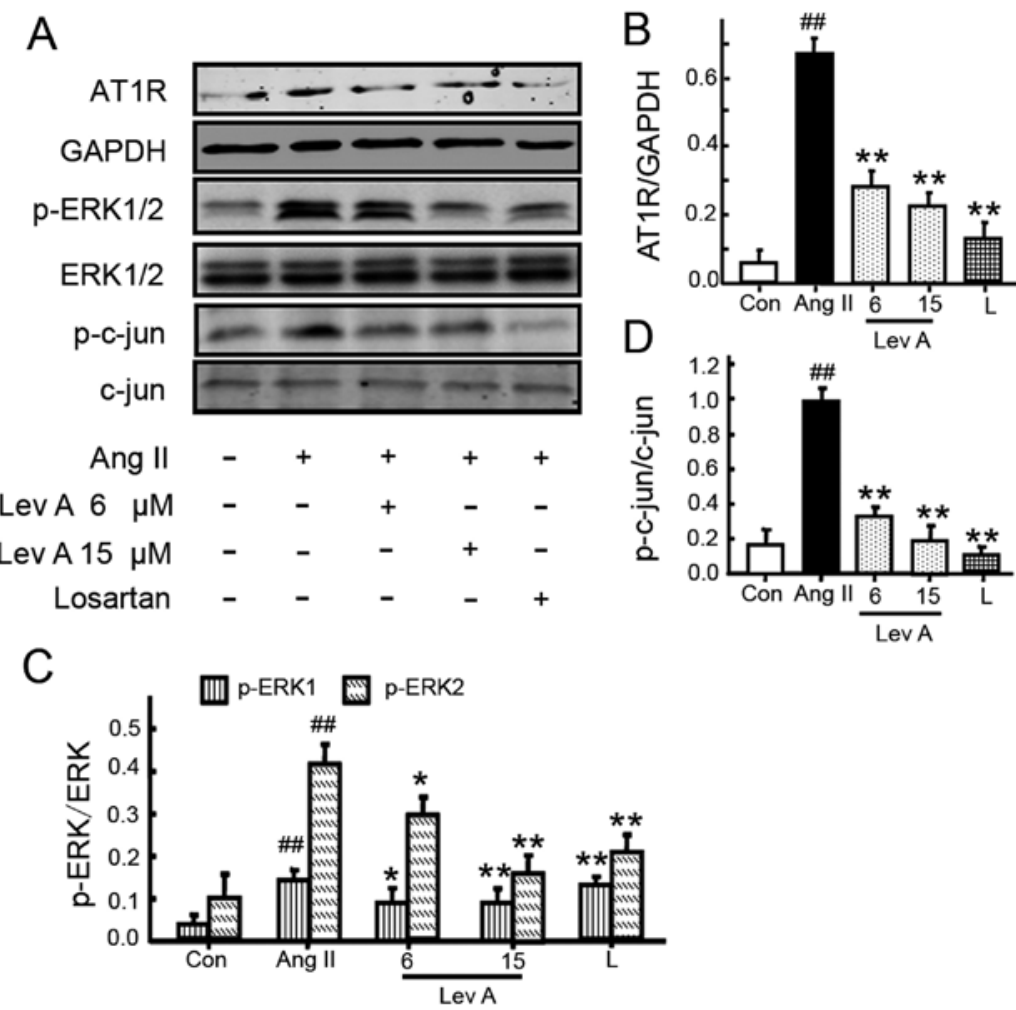

Figure 3. Effect of Lev A on Ang II-induced RAS molecule expression in vitro. (A) Effect of Lev A on the expression of AT1R and phosphorylation of ERK and c-Jun. (B-D) Quantitative data of AT1R, p-ERK and p-c-Jun, respectively. HSCs were pre-treated with Lev $(6$ or $15 \mu \mathrm{M})$ or losartan $(1 \mu \mathrm{M})$ for $2 \mathrm{~h}$, then treated with Ang II $(0.1 \mu \mathrm{M})$ for different durations. Total protein was isolated and subjected to western blot analysis. Each bar represents the mean \pm standard deviation of three independent experiments. ${ }^{\# \#} \mathrm{P}<0.01$ vs. control group; ${ }^{*} \mathrm{P}<0.05,{ }^{* *} \mathrm{P}<0.01$ vs. Ang II group. Lev A, levistilide A; Ang II, angiotensin II; RAS, renin angiotensin system; AT1R, type 1 angiotensin receptor; p-, phosphorylated; HSCs, hepatic stellate cells; Con, control; L, losartan.

A

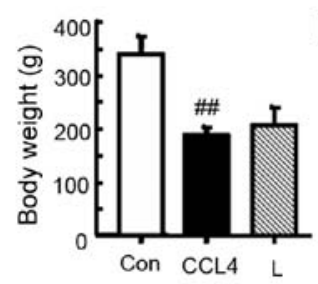

E

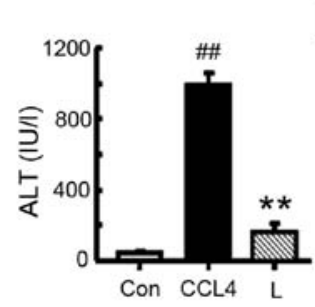

B

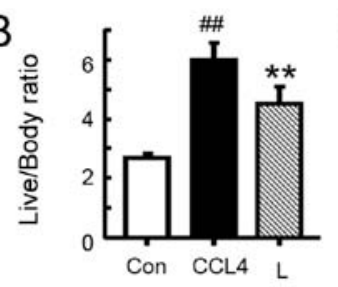

$\mathrm{F}$

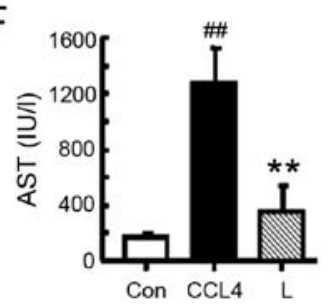

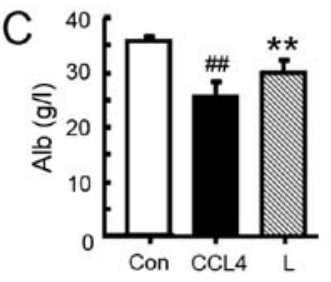

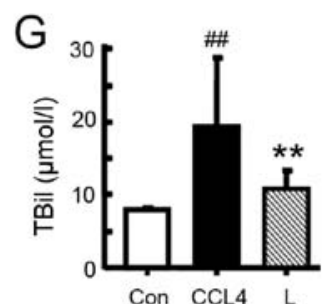

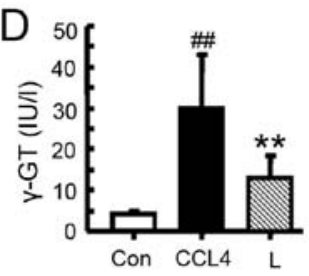

$\mathrm{H}$

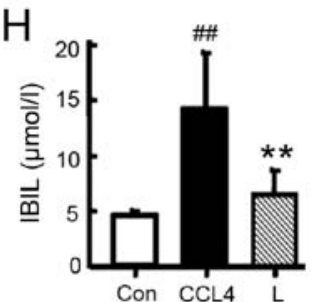

I
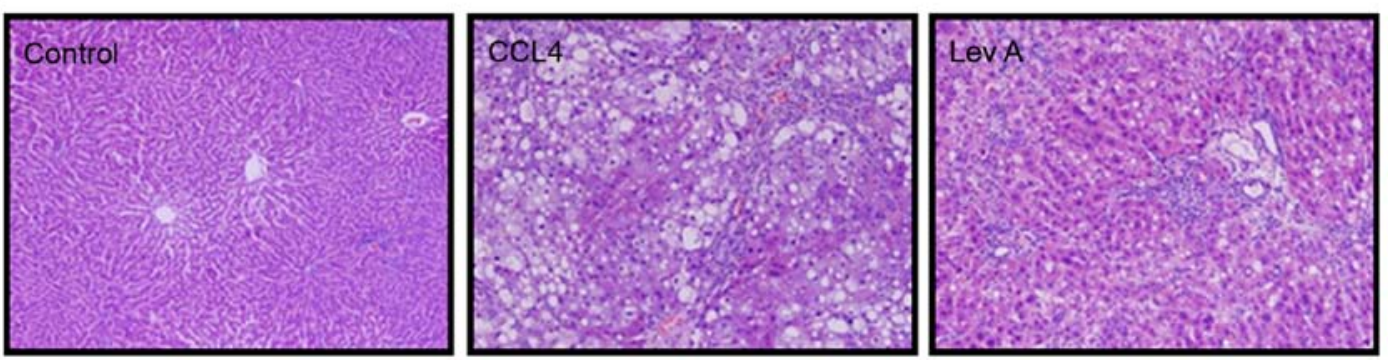

Figure 4. Effect of Lev A on the body weight and liver function of $\mathrm{CCL}_{4}$-treated rats. (A) Body weight and (B) liver/body weight ratio. Levels of (C) Alb, (D) $\gamma$-GT, (E) ALT, (F) AST, (G) TBiL and (H) IBIL. (I) H\&E staining of the rat liver (original magnification, x200). Each bar represents the means \pm standard deviation $(\mathrm{n}=8) .{ }^{\# /} \mathrm{P}<0.01$ vs. control group; ${ }^{* *} \mathrm{P}<0.01$ vs. model group. Lev A, levistilide A; Alb, plasma albumin; $\gamma$-GT, $\gamma$-glutamyl transpeptidase; ALT, alanine aminotransferase; AST, aspartate aminotransferase; TBiL, total bilirubin; IBIL, indirect bilirubin; H\&E, hematoxylin and eosin; Con, control; L, losartan. 


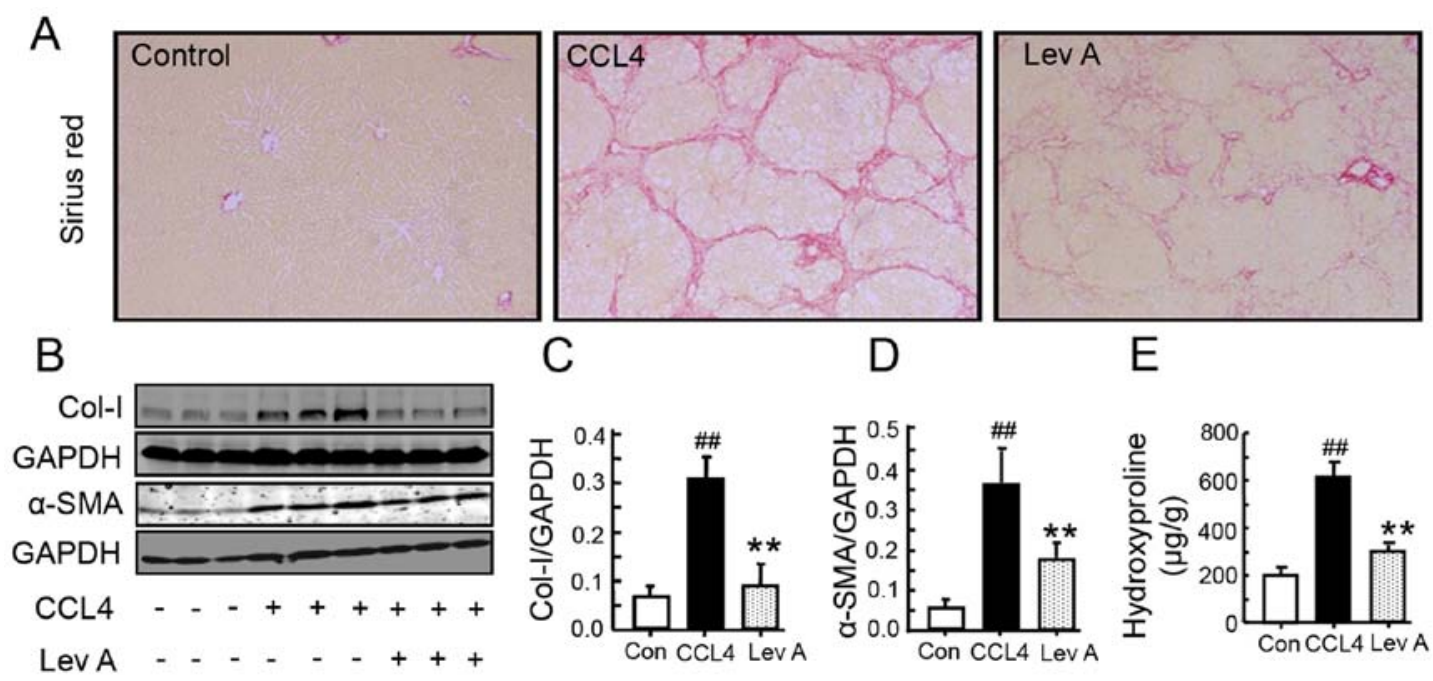

Figure 5. Effect of Lev A on CCL4-induced rat hepatic fibrosis. (A) Collagen deposition was determined by sirius red staining (magnification, x200). (B) The expression of $\alpha$-SMA and collagen I protein was analyzed by western blotting. (C and D) Quantification of $\alpha$-SMA and collagen I. (E) Total collagen as determined by hydroxyproline analysis. Each bar represents the means \pm standard deviation $(n=8) .{ }^{\# \#} \mathrm{P}<0.01$ vs. control group; ${ }^{* *} \mathrm{P}<0.01$ vs. model group. Lev A, levistilide A; $\alpha$-SMA, smooth muscle $\alpha$-actin; Con, control.
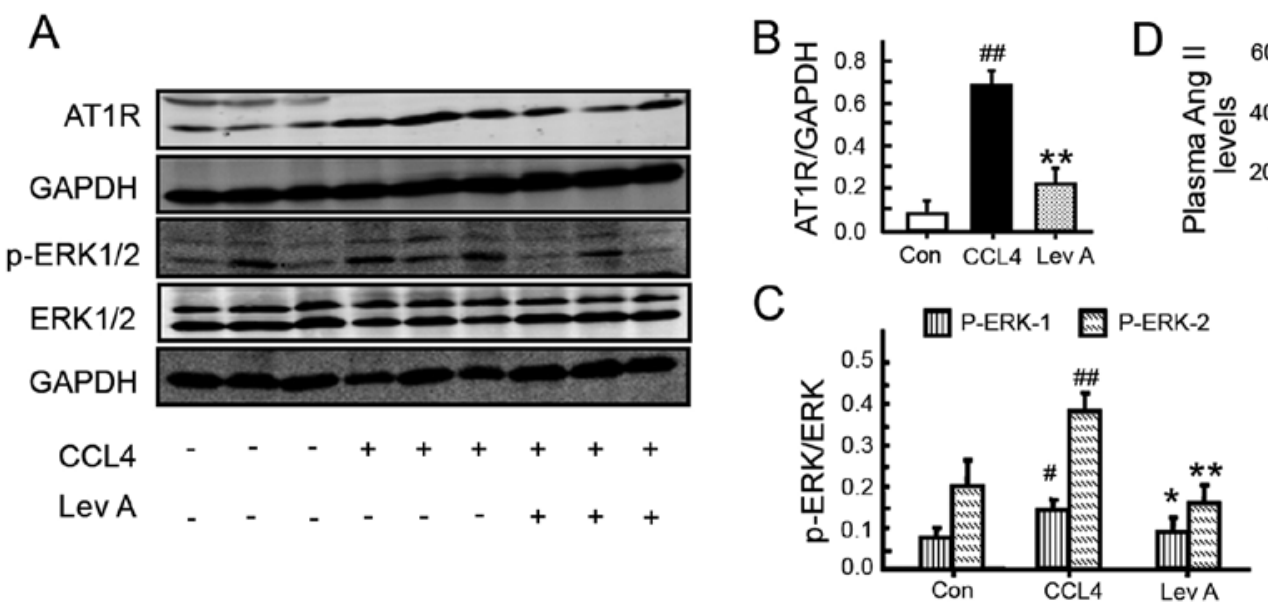

Figure 6. Effect of Lev A on the molecules of RAS in vivo. (A) The expression of AT1R, p-ERK1/2 and ERK1/2 in rat liver. (B and C) Relative levels of AT1R and p-ERK1/2, respectively. (D) Plasma Ang II levels determined by radioimmunoassay. Each bar represents the means \pm standard deviation (n=8). ${ }^{\#} \mathrm{P}<0.05$, ${ }^{\# \#} \mathrm{P}<0.01$ vs. control group; ${ }^{\mathrm{P}}<0.05,{ }^{* *} \mathrm{P}<0.01$ vs. model group. Lev A, levistilide A; RAS, renin angiotensin system; AT1R, type 1 angiotensin receptor; p-, phosphorylated; Ang II, angiotensin II; Con, control.

that Lev A is a good candidate for the treatment of hepatic fibrosis.

It has been widely recognized that the profibrotic activity of Ang II is mainly mediated by AT1R (25-27). Consistent with previous studies (11-13), the present study also revealed that the AT1R levels were significantly elevated in Ang II-treated HSCs and $\mathrm{CCL}_{4}$-treated rat liver. Lev A treatment inhibited Ang II- and $\mathrm{CCL}_{4}$-induced AT1R upregulation in HSCs and in the rat liver. Following binding to AT1R, Ang II also activates intracellular signaling pathways, including MAPKs and transcription factor AP-1 $(12,28)$. Ang II induces the phosphorylation of ERK1/2 and activation of AP-1, and subsequently increases the expression of procollagen $\alpha 1$ and TGF- $\beta 1$ in HSCs $(12,25,29)$. The results of the present study revealed that Lev A treatment significantly suppressed Ang II-induced phosphorylation of ERK1/2 and c-Jun in HSCs. Lev A also inhibited $\mathrm{CCL}_{4}$-induced ERK and
c-Jun phosphorylation in rat livers. These results suggested that Lev A may exert its anti-hepatic fibrosis effect by inhibiting Ang II-AT1R signaling and consequently suppressing ERK/c-Jun activation. In addition to the ACE-Ang II-AT1R pathway, the ACE2-Ang 1-7-Mas axis also serves an important role in hepatic fibrosis. ACE2 can degrade Ang II to Ang 1-7, an important, biologically active component of this system, which exerts opposite effects to those of Ang II through its receptor, Mas $(30,31)$. The ACE2-Ang 1-7-Mas axis counteracts the effects of the ACE-Ang II-AT 1R axis in hepatic fibrosis $(30,32)$. Ang 1-7 has shown antifibrotic actions in the bile-duct-ligated rat (33-35). Blockade of the Ang 1-7 receptor has been demonstrated to aggravate liver fibrosis (36). It has been reported that losartan may upregulate the cardiac ACE2-Ang(1-7)-Mas axis expression (37). Therefore, the role of Lev A on the ACE2-Ang(1-7)-Mas axis should be further investigated. 
In summary, the present study demonstrated that Lev A is able to inhibit Ang II-induced HSC activation and improve experimental hepatic fibrosis induced by $\mathrm{CCL}_{4}$. Lev A inhibits the upregulation of AT1R and activation of ERK1/2 and c-Jun in vitro and in vivo. As a limitation of the present study, the protein was extracted from liver tissues, not from isolated HSC cells. Our previous study (21) demonstrated that swertiamarin is able to attenuate experimental liver fibrosis by regulating the Ang II/AT1R pathway. The results of the current study also indicated that Lev $\mathrm{A}$ is a potential agent for the treatment of hepatic fibrosis by suppressing the activation of Ang II/AT1R/ERK/c-Jun signaling in HSCs. These results imply that the Ang II/AT1R pathway is an important target for the screening of optimal agents for reversing liver fibrosis from Chinese herbs.

\section{Acknowledgements}

Not applicable.

\section{Funding}

The present study was supported by grants from the National Natural Science Foundation of China (grant nos. 81603458, 81730109 and 81473479), Special Program for Traditional Chinese Medicine Research of Shanghai Municipal Commission of Health and Family Planning (grant no. 2016JQ001), Baoshan Branch, Shuguang Hospital Affiliated to Shanghai University of Traditional Chinese Medicine (grant no. GZRPYZZ-201603), Construction plan of medical specialty in Baoshan District of Shanghai (grant no. BSZK-2018-A03), National Science and Technology Major Project (grant no. 2014ZX10005001) and 'Three-Year Action Plan' for Development of TCM in Shanghai (grant no. 16CR1026B).

\section{Availability of data and materials}

The datasets used and/or analyzed during the current study are available from the corresponding author on reasonable request.

\section{Authors' contributions}

ShuaL and CL were involved in conception and design of the study. ShuL, WZ and ZZ performed the experiments. ShuL, BC and ShuaL analyzed the data. ShuL and BC wrote the manuscript. All authors read and approved the final manuscript.

\section{Ethics approval and consent to participate}

The protocols were approved by the Committee on the Ethics of Animal Experiments of Shanghai University of Traditional Chinese Medicine, China. All animals received humane care during the study.

\section{Patient consent for publication}

Not applicable.

\section{Competing interests}

The authors declare that they have no competing interests.

\section{References}

1. Khomich O, Ivanov AV and Bartosch B: Metabolic hallmarks of hepatic stellate cells in liver fibrosis. Cells 9: pii: E24, 2019.

2. Huang Y, Deng X and Liang J: Modulation of hepatic stellate cells and reversibility of hepatic fibrosis. Exp Cell Res 352: 420-426, 2017.

3. Cai X, Wang J, Zhou Q, Yang B, He Q and Weng Q: Intercellular crosstalk of hepatic stellate cells in liver fibrosis: New insights into therapy. Pharmacol Res 155: 104720, 2020.

4. Sancho-Bru P and Ginès P: Targeting the renin-angiotensin system in liver fibrosis. Hepatol Int 10: 730-732, 2016.

5. Li S, Zhao W, Tao Y and Liu C: Fugan Wan alleviates hepatic fibrosis by inhibiting ACE/Ang II/AT-1R signaling pathway and enhancing ACE2/Ang 1-7/Mas signaling pathway in hepatic fibrosis rat models. Am J Transl Res 12: 592-601, 2020.

6. Bataller R, Ginès P, Nicolás JM, Görbig MN, Garcia-Ramallo E, Gasull X, Bosch J, Arroyo V and Rodés J: Angiotensin II induces contraction and proliferation of human hepatic stellate cells. Gastroenterology 118: 1149-1156, 2000.

7. Bataller R, Schwabe RF, Choi YH, Yang L, Paik YH, Lindquist J, Qian T, Schoonhoven R, Hagedorn CH, Lemasters JJ and Brenner DA: NADPH oxidase signal transduces angiotensin II in hepatic stellate cells and is critical in hepatic fibrosis. J Clin Invest 112: 1383-1394, 2003.

8. Saber S, Goda R, El-Tanbouly GS and Ezzat D: Lisinopril inhibits nuclear transcription factor kappa B and augments sensitivity to silymarin in experimental liver fibrosis. Int Immunopharmacol 64: 340-349, 2018.

9. Czechowska G, Celinski K, Korolczuk A, Wojcicka G, Dudka J, Bojarska A, Madro A and Brzozowski T: The effect of the angiotensin II receptor, type 1 receptor antagonists, losartan and telmisartan, on thioacetamide-induced liver fibrosis in rats. J Physiol Pharmacol 67: 575-586, 2016.

10. Kim MY, Baik SK, Park DH, Jang YO, Suk KT, Yea CJ, Lee IY, Kim JW, Kim HS, Kwon SO, et al: Angiotensin receptor blockers are superior to angiotensin-converting enzyme inhibitors in the suppression of hepatic fibrosis in a bile duct-ligated rat model. J Gastroenterol 43: 889-896, 2008.

11. Reza HM, Tabassum N, Sagor MA, Chowdhury MR, Rahman M, Jain P and Alam MA: Angiotensin-converting enzyme inhibitor prevents oxidative stress, inflammation, and fibrosis in carbon tetrachloride-treated rat liver. Toxicol Mech Methods 26: 46-53, 2016.

12. Bataller R, Gäbele E, Schoonhoven R, Morris T, Lehnert M, Yang L, Brenner DA and Rippe RA: Prolonged infusion of angiotensin II into normal rats induces stellate cell activation and proinflammatory events in liver. Am J Physiol Gastrointest Liver Physiol 285: G642-G651, 2003.

13. Huang Y, Li Y, Lou A, Wang GZ, Hu Y, Zhang Y, Huang W, Wang J, Li Y, Zhu X, et al: Alamandine attenuates hepatic fibrosis by regulating autophagy induced by NOX4-dependent ROS. Clin Sci (Lond) 134: 853-869, 2020.

14. Wei H, Lu H, Li D, Zhan Y, Wang Z and Huang X: The expression of AT1 receptor on hepatic stellate cells in rat fibrosis induced by CCl4. Chin Med J (Engl) 114: 583-587, 2001.

15. Wang K, Cao P, Wang H, Tang Z, Wang N, Wang J and Zhang Y: Chronic administration of Angelica sinensis polysaccharide effectively improves fatty liver and glucose homeostasis in high-fat diet-fed mice. Sci Rep 6: 26229, 2016.

16. Pu X, Fan W, Yu S, Li Y, Ma X, Liu L, Ren J and Zhang W: Polysaccharides from Angelica and Astragalus exert hepatoprotective effects against carbon-tetrachloride-induced intoxication in mice. Can J Physiol Pharmacol 93: 39-43, 2015.

17. Lee TF, Lin YL and Huang YT: Studies on antiproliferative effects of phthalides from Ligusticum chuanxiong in hepatic stellate cells. Planta Med 73: 527-534, 2007.

18. Friedman SL, Rockey DC, McGuire RF, Maher JJ, Boyles JK and Yamasaki G: Isolated hepatic lipocytes and Kupffer cells from normal human liver: Morphological and functional characteristics in primary culture. Hepatology 15: 234-243, 1992.

19. Sohail MA, Hashmi AZ, Hakim W, Watanabe A, Zipprich A, Groszmann RJ, Dranoff JA, Torok NJ and Mehal WZ: Adenosine induces loss of actin stress fibers and inhibits contraction in hepatic stellate cells via Rho inhibition. Hepatology 49: 185-194, 2009. 
20. Ala-Kokko L, Pihlajaniemi T, Myers JC, Kivirikko KI and Savolainen ER: Gene expression of type I, III and IV collagens in hepatic fibrosis induced by dimethylnitrosamine in the rat. Biochem J 244: 75-79, 1987.

21. Li S, Wang Q, Tao Y and Liu C: Swertiamarin attenuates experimental rat hepatic fibrosis by suppressing angiotensin II-angiotensin type 1 receptor-extracellular signal-regulated kinase signaling. J Pharmacol Exp Ther 359: 247-255, 2016.

22. Wei HS, Lu HM, Li DG, Zhan YT, Wang ZR, Huang X, Cheng JL and Xu QF: The regulatory role of AT 1 receptor on activated HSCs in hepatic fibrogenesis:Effects of RAS inhibitors on hepatic fibrosis induced by $\mathrm{CCl}(4)$. World J Gastroenterol 6: 824-828, 2000.

23. Wu Y, Li Z, Wang S, Xiu A and Zhang C: Carvedilol inhibits angiotensin II-induced proliferation and contraction in hepatic stellate cells through the RhoA/Rho-kinase pathway. Biomed Res Int 2019: 7932046, 2019.

24. Zhang X, Zhang F, Kong D, Wu X, Lian N, Chen L, Lu Y and Zheng S: Tetramethylpyrazine inhibits angiotensin II-induced activation of hepatic stellate cells associated with interference of platelet-derived growth factor $\beta$ receptor pathways. FEBS J 281: 2754-2768, 2014.

25. Li X, Meng Y, Wu P, Zhang Z and Yang X: Angiotensin II and aldosterone stimulating NF-kappaB and AP-1 activation in hepatic fibrosis of rat. Regul Pept 138: 15-25, 2007.

26. Yang L, Bataller R, Dulyx J, Coffman TM, Ginès P, Rippe RA and Brenner DA: Attenuated hepatic inflammation and fibrosis in angiotensin type 1a receptor deficient mice. J Hepatol 43: 317-323, 2005

27. Wang XN, Hu YY, Liu CH, Liu P and Zhu DY: Effects of salvianolic acid $\mathrm{B}$ on expressions of TGF-betal and its receptors in liver of rats with dimethylnitrosamine-induced hepatic fibrosis. Zhong Xi Yi Jie He Xue Bao 3: 286-289, 2005 (In Chinese).

28. Ruiz-Ortega M, Lorenzo O, Rupérez M, Suzuki Y and Egido J: Angiotensin II activates nuclear transcription factor-kappaB in aorta of normal rats and in vascular smooth muscle cells of AT1 knockout mice. Nephrol Dial Transplant 16 (Suppl 1): S27-S33, 2001.

29. Vogt PK: Jun, the oncoprotein. Oncogene 20: 2365-2377, 2001.

30. Donoghue M, Hsieh F, Baronas E, Godbout K, Gosselin M Stagliano N, Donovan M, Woolf B, Robison K, Jeyaseelan R, et al: A novel angiotensin-converting enzyme-related carboxypeptidase (ACE2) converts angiotensin I to angiotensin 1-9. Circ Res 87: E1-E9, 2000.
31. Tipnis SR, Hooper NM, Hyde R, Karran E, Christie G and Turner AJ: A human homolog of angiotensin-converting enzyme. Cloning and functional expression as a captoprilinsensitive carboxypeptidase. J Biol Chem 275: 33238-33243, 2000 .

32. Santos RA, Ferreira AJ, Verano-Braga $\mathrm{T}$ and Bader $\mathrm{M}$ : Angiotensin-converting enzyme 2, angiotensin-(1-7) and Mas: New players of the renin-angiotensin system. J Endocrinol 216: R1-R17, 2013.

33. Lubel JS, Herath CB, Tchongue J, Grace J, Jia Z, Spencer K, Casley D, Crowley P, Sievert W, Burrell LM and Angus PW: Angiotensin-(1-7), an alternative metabolite of the renin-angiotensin system, is up-regulated in human liver disease and has antifibrotic activity in the bile-duct-ligated rat. Clin Sci (Lond) 117: 375-386, 2009.

34. Dasuri K, Zhang L and Keller JN: Oxidative stress, neurodegeneration, and the balance of protein degradation and protein synthesis. Free Radic Biol Med 62: 170-185, 2013.

35. Herath CB, Warner FJ, Lubel JS, Dean RG, Jia Z, Lew RA, Smith AI, Burrell LM and Angus PW: Upregulation of hepatic angiotensin-converting enzyme 2 (ACE2) and angiotensin-(1-7) levels in experimental biliary fibrosis. J Hepatol 47: 387-395, 2007.

36. Pereira RM, Dos Santos RA, Teixeira MM, Leite VH, Costa LP, da Costa Dias FL, Barcelos LS, Collares GB and Simões e Silva AC: The renin-angiotensin system in a rat model of hepatic fibrosis: Evidence for a protective role of Angiotensin-(1-7). J Hepatol 46: 674-681, 2007.

37. Wang X, Ye Y, Gong H, Wu J, Yuan J, Wang S, Yin P, Ding Z, Kang L, Jiang Q, et al: The effects of different angiotensin II type 1 receptor blockers on the regulation of the ACE-AngII-AT1 and ACE2-Ang(1-7)-Mas axes in pressure overload-induced cardiac remodeling in male mice. J Mol Cell Cardiol 97: 180-190, 2016.

This work is licensed under a Creative Common Attribution-NonCommercial-NoDerivatives 4.0 International (CC BY-NC-ND 4.0) License. 